Originally published in: Martin-Martinez, J. ; Diaz, J. ; Rodriguez, R. ; Nafria, M. ; Aymerich, X. ; Roca, E. ; Fernandez, F.V.; Rubio, A Characterization of random telegraph noise and its impact on reliability of SRAM sense amplifiers In: CMOS Variability (VARI), 2014 5th European Workshop on. Publication Year: 2014 , Page(s): $1-6$.

\title{
Characterization of Random Telegraph Noise and its impact on reliability of SRAM sense amplifiers
}

\author{
J. Martin-Martinez ${ }^{1}$, J. Diaz ${ }^{1}$, R. Rodriguez ${ }^{1}$, M. Nafria ${ }^{1}$, X. Aymerich ${ }^{1}$, E. Roca ${ }^{2}$, F.V. Fernandez ${ }^{2}$, A. Rubio ${ }^{3}$ \\ ${ }^{1}$ Departament d'Enginyeria Electrònica, Universitat Autònoma de Barcelona (UAB), Bellaterra, Spain \\ ${ }^{2}$ Instituto de Microelectrónica de Sevilla, CSIC and Universidad de Sevilla \\ ${ }^{3}$ Departament d'Enginyeria Electrònica, Universitat Politècnica de Catalunya (UPC), Barcelona \\ *Corresponding author: Javier.martin.martinez@uab.es
}

A new method for the analysis of multilevel Random Telegraph Noise (RTN) signals has been recently presented, which can also be applied in the case of large background noise. In this work, the method is extended to evaluate the RTN-related variation of the device drain current. The RTN parameters obtained from experimental traces are used to simulate the impact of RTN in the drain current of pMOS transistors in SRAM voltage sense amplifiers. The results show that RTN can lead to read errors of the stored data.

\section{INTRODUCTION}

RTN has attracted much attention as a temporal statistically independent variation enlarged with scaling in image sensors [1], flash memories [2] and SRAMs [3]. In relation with SRAMs, one of the more critical components in embedded high performance processors, a significant write-margin degradation has been recently reported in [4]. In this work we investigate the impact of RTN on the robustness of a voltage Sense Amplifier of SRAMs, showing also reliability degradation in the reading cycles. The RTN signals that are introduced in the drain current of the pMOS transistors have been generated using (statistical) parameters that are experimentally determined using a recently developed method for the characterization of RTN signals [5]. The method is extended to evaluate the fluctuation limits of the drain current in the device.

\section{Characterizing RANDOM TELEGRAPH Signals}

In MOSFETs, Random Telegraph Noise has been associated to the trapping/detrapping of charges in/from defects in the device. These defects can be inherent to the fabrication process or created by aging mechanisms triggered during device operation [6]. RTN is a feature of ultrascaled devices, whose very reduced active area allows resolving the individual fluctuators. As an example, Fig.1 illustrates a typical RTN signal, in this case mathematically built.

When the background noise is relevant when compared with the current/voltage steps in the RTN, the precise characterization can be difficult, especially in complex RTN. To overcome this problem, the Weighted Time Lag method (wTL) [5] has been recently reported. However, from a circuit point of view, it is important to accurately determine the amplitudes of current fluctuations associated to RTN, in order to establish precise safety margins for the circuit operation. In the next section, we will show how the wTL method can also be useful to evaluate these current fluctuations.

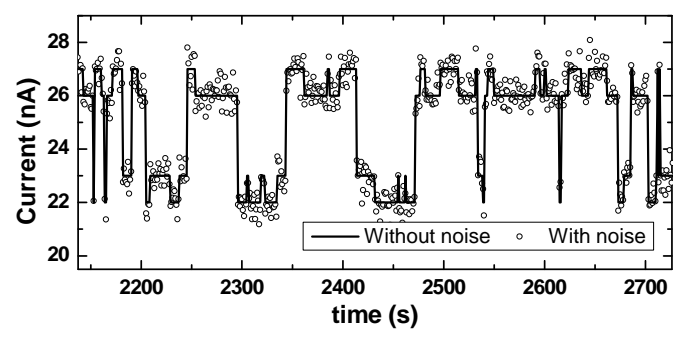

Fig. 1. Monte Carlo generated RTN which results from the charge fluctuations in two defects, so that four different current levels (black line) are observed. Gaussian noise with $\sigma=0.4 \mathrm{nA}$ has been added to the RTN to reproduce the background noise (open circles).

\section{EVALUATING THE AMLITUDE OF THE RTN DRAIN CURRENT FLUCTUATIONS}

To show the procedure to obtain the amplitude of the drain current fluctuation related to the RTN, by applying the wTL method to the analysis of RTN signals, first, the RTN in Fig. 1 will be used as an example and, afterwards, RTN coming from experimental measurements in pMOS transistors will be studied. The histogram of the RTN currents in Fig. 1 is plotted in Fig. 2 (bars). Since we are dealing with a simulated RTN, it is straightforward to deduce the analytical equation that describes this histogram. For each of the current levels of the generated RTN, a Gaussian distribution is built, with average $I_{L i}$ (the value of each current level). The standard deviation is the same for all the distributions and equal to the standard deviation considered for the background noise, i.e., $\sigma=0.4 \mathrm{nA}$. The height of each Gaussian distribution is proportional to the probability of occurrence of the associated current level. These probabilities can be easily calculated from the (known, in this case) input parameters used to generate the RTN. Therefore, the analytical probability density function obeys eq. 1 :

$$
F(I)=\frac{K}{2 \pi \sigma^{2}} \sum_{i=1}^{L} A_{i} \exp \left(\frac{-\left(I-I_{L, i}\right)^{2}}{2 \sigma^{2}}\right)
$$

being $L$ the number of levels in the RTN, $i$ an index that denotes the level, $I$ the current at each point of the RTN, $\sigma$ the standard deviation of the background noise, $A_{i}$ the peak height and $I_{L, i}$ the current value for each RTN level. ' $K$ ' is a normalization constant. For coherency with the wTL method, the value of $K$ has been chosen so that the maximum of $F(I)$ is 1. The dots in Fig. 2 corresponds to the analytical probability distribution obtained from eq. 2. But in a realistic case, the parameters of the best fit Gaussian distributions are unknown, 
so $F(I)$ cannot be built. The wTL method, however, can be applied to fit the current histogram. To show this point, Fig. 3 shows the $\Psi$ function obtained from the wTL method for $\alpha=$ $\sigma$, from this figure we can obtain the diagonal of the $\Psi$ function (dashed black line), which is indicated in Fig. 2 with a red line. From now on, we will name this function as 'the wTL $\Psi$ diagonal function'. Note the accurate match between the wTL $\Psi$ diagonal function and the RTN histogram is obtained for $\alpha=\sigma$.

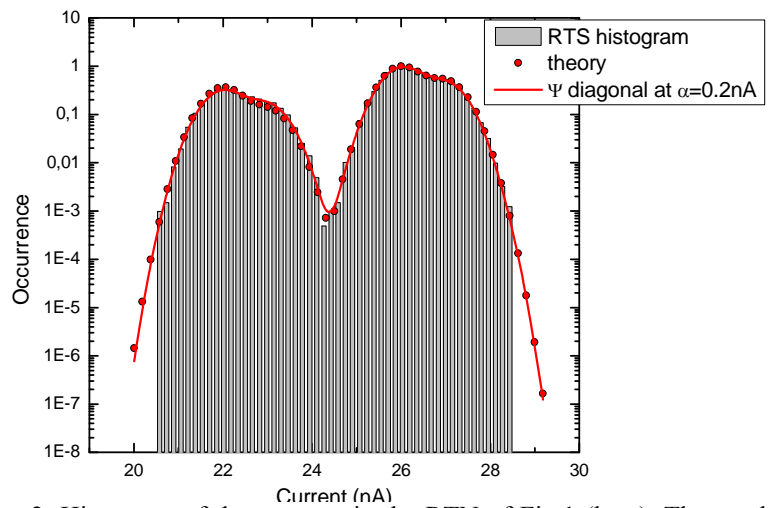

Fig. 2. Histogram of the currents in the RTN of Fig.1 (bars). The symbols corresponds to the theoretical $F(I)$ function (eq 2). The red line shows the wTL $\Psi$ diagonal function with $\alpha=0.4 \mathrm{nA}$.

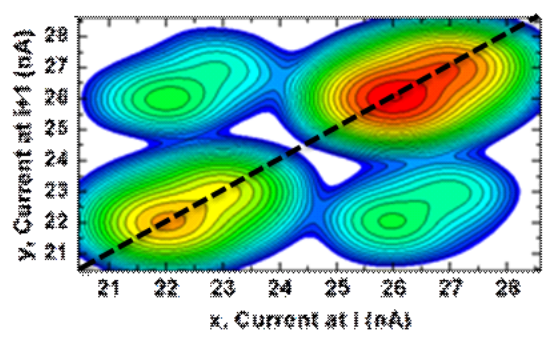

Fig. 3. Plot of the $\Psi(\mathrm{x}, \mathrm{y})$ function for the RTN in Fig. 2 for $\alpha=0.4 \mathrm{nA}$.

Since $\sigma$ is unknown in experimental RTN traces, these results indicates that $\sigma$ can be calculated using the wTL method, as the $\alpha$ value of the wTL $\Psi$ diagonal function that better fits the RTN histogram. Additionally, the wTL $\Psi$ diagonal function with $\alpha=\sigma$ (red circles) also matches the analytical probability density function (red line), even outside of the histogram limits. This leads to a second conclusion: the wTL $\Psi$ diagonal function that better fits the RTN histogram can be used to evaluate the amplitude of the drain current fluctuations in the device, even outside the experimental window, that is, for longer operation times.

\section{EXPERIMENTAL RESULTS IN PMOS TRANSISTORS.}

In the following, the methodology presented in section III will be applied to determine the amplitude of the drain current fluctuations from RTN traces experimentally obtained, where all the RTN parameters are unknown (Fig. 4). The discrete sudden changes in the current reveal the presence of defects in the device, leading to RTN in the drain current.

The wTL $\Psi$ diagonal function has been calculated for a wide range of $\alpha$ values, and the difference between this function and the histogram (the error) has been calculated. Fig. 5 shows the envelope of the histogram for the RTN of Fig. 4 (dots) and the wTL $\Psi$ diagonal function that better fits this histogram (line). Extrapolating the wTL $\Psi$ diagonal function to low occurrence probabilities provides the information about the lower and upper limits expected from the RTN currents for large operation times of the transistors. Note that the function is very steep, leading to well defined limits for the drain current.

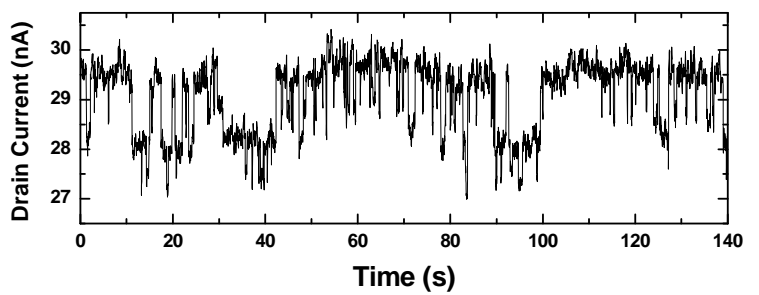

Fig. 4. RTN traces measured in the drain current of a high-k/metal gate pMOS transistor. Drain voltage $-200 \mathrm{mV}$ and gate voltage $-0.6 \mathrm{~V}$

\section{IMPACT OF RTN I SRAM SENSE AMPLIFIERS}

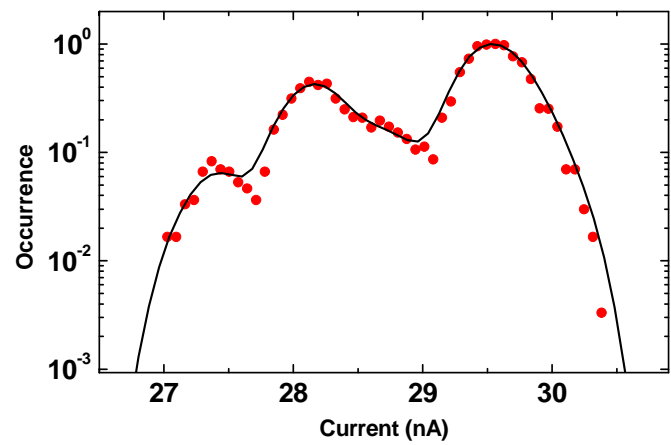

Fig. 5. Current histograms of the RTN traces in Fig. 5 (symbols) and their best fit wTL $\Psi$ diagonal function (lines). The extrapolation to low probability occurrence allows determining the upper and lower bounds of the drain current.

We analyse in this section how RTN may affect the robustness of a Sense Amplifier (SA) circuit, because they are key components of memory systems affecting its reliability and performances. Fig. 6 shows the circuit, a conventional voltage sense amplifier receiving signals 'bit' and 'bit b' (negate) from the column cells matrix. The SA 'sense' output signal is regenerated through a threshold gate (inverter). It is considered a hypothetical situation in which the bit lines have been precharged to a given voltage and an SRAM cell storing a logical " 0 " is read. Then, a small imbalance of $50 \mathrm{mV}$ is produced at the bit lines (inputs of the sense amplifier in Fig. $6)$. The output of the sense amplifier should change to a low state, providing a logical " 0 " at its output. Although the plot in Fig. 4 was obtained for fluctuations of the drain current, this is not an appropriate characterization for circuit simulation purposes. Therefore, the changes in the drain current due to RTN have been mapped to variations in the threshold voltages of the transistors. 


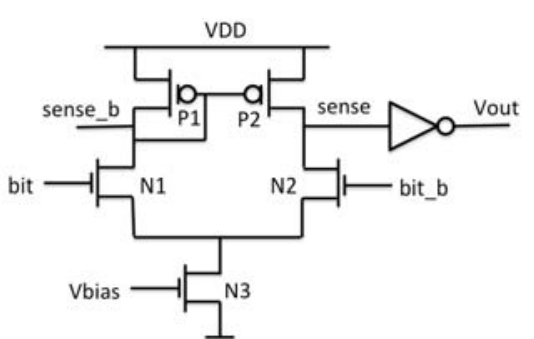

Fig. 6. Sense amplifier scheme. RTN variations of the drain current are only considered in the pMOS devices

The circuit was simulated for all possible deviations of the threshold voltages of the three PMOS transistors of the circuit in Fig. 6 (two pMOS transistors, P1 and P2, from the SA core and one, P3, from the output inverter). As the level of the RTN traces when a sensing operation is performed is unknown, a statistical study with a total of 20,000 samples has been performed. The results show that in 129 situations the circuit output is not correct due to the RTN. Figure 7 shows the 20,000 simulations as a function of the variations incorporated in the threshold voltage of the three pMOS transistors. Green points indicate situations where the circuit output voltage is correct, whereas red points (129 points) are those where the circuit output voltage is not correct. It can be seen that incorrect decisions are taken only when certain combinations of the variations of the three threshold voltages occur. Figure 8 illustrates the transient evolution of the output voltage for three different situations when a transition from a high state to a low state is expected. The graph in black corresponds to one of the green points in Fig. 7 whose behaviour is not affected by RTN, showing a sharp transition to low state. The green

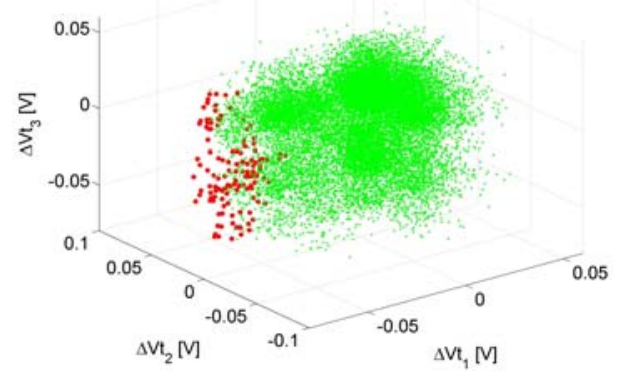

Fig. 7 Values of the threshold voltage variations for transistors P1, P2 and P3 of the sense amplifier due to RTN for 20,000 simulations. In red, simulations where the circuit does not take the correct decision, and, in green, simulations where the circuit functions correctly.

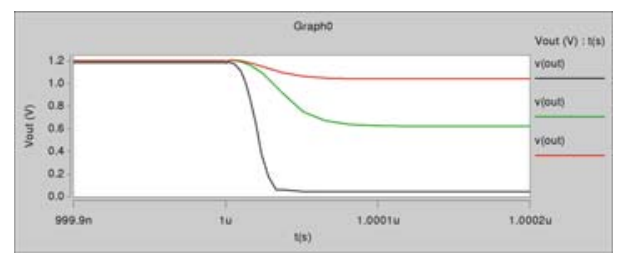

Fig. 8. Transient evolution of the output voltage of the sense amplifier when a transition to a logical " 0 " is expected and three different RTN values. The graph in black shows a typical correct behavior, despite the RTN, whereas, the other graphs (green and red) show two situations where the circuit output is affected by the RTN, taking an incorrect output value. and red graphs correspond to two different red points in Fig. 7 where it can clearly be observed that the output remains at a high voltage value in one case (red graph) and at an intermediate voltage in the other (green graph). In none of the cases a logical " 0 " is provided at the output.

\section{CONCLUSIONS}

A recently presented method for the analysis of Random Telegraph Noise (RTN) signals in the presence of large background noise has been extended to evaluate the limits of variation of the drain current related to RTN. The theoretical framework is presented and applied to the analysis of experimental signals in pMOS transistors. It is shown that the envelope of the current histograms coming from RTN can be accurately fitted with only one parameter, which is indicative of the standard deviation of the background noise. The method is used to evaluate the characteristic parameters of RTN signals measured on pMOS devices, from which the amplitude of the drain current fluctuations in the transistors is determined. The extracted parameters are used to generate RTN drain current signals which, in order to be introduced in a circuit simulator are transformed into RTN threshold voltage signals. The impact of the pMOS threshold voltage random signals on SRAM sense amplifiers is evaluated. Monte Carlo simulations of the circuit demonstrate that, because of RTN, some particular combinations of pMOS threshold voltages can lead to errors in the read operation of the cell.

\section{ACKNOWLEDGMENTS}

This work has been partially funded by the Spanish MINECO and ERDF (TEC2008-01856 TEC2010-16126, TEC2010-14825, TEC2013-45638-C3-R), the Generalitat de Catalunya (2014 SGR 384, 2014SGR-455) and the Junta de Andalucía (P12-TIC-1481).

\section{REFERENCES}

[1] Jun-Myung Woo, Hong-Hyun Park, Hong Shick Min, Young June Park, Sung-Min Hong, and Chan Hyeong Park. Statistical analysis of random telegraph noise in cmos image sensors. In Simulation of Semiconductor Processes and Devices, 2008. SISPAD 2008. International Conference on, pp. 77-80, 9-11 2008.

[2] H. Kurata, K. Otsuga, S. Kaiiyama, T.Osabe, Y. Sasago, S. Narumi, K. Tokami, S. Kamohara, and O. Tsuchiya. Random telegraph signal in flash memory: Its impact on scaling of multilevel flash memory beyond the 90-nm node. Solid-State Circuits, IEEE Journal of, Vol. 42, No. 6, pp. 1362-1369, jun., 2007.

[3] M. Tanizawa, S. Ohbayashi, T. Okagaki, K. Sonoda, K. Eikyu, Y. Hirano, K. Ishikawa, O. Tsuchiya, and Y. Inoue. Application of a statistical compact model for random telegraph noise to scaled-sram vmin analysis. In VLSI Technology, 2010 Symposium on, pp. 95-96, 16-18 2010.

[4] S.O.Toh et al.,'Imapct of random telegraph signals in 45nm SRAM", in IEDM Tech. Dig, 2009, pp. 767-770.

[5] J. Martin-Martinez, J. Diaz, R. Rodriguez, M. Nafria and X. Aymerich, "New Weighted Time Lag Method for the Analysis of Random Telegraph Signals", IEEE Electron Device Letters, vol 35, no 4, pp. 479-48 (2014).

[6] M. Toledano-Luque et. al. "Degradation of time dependent variability due to interface state generation" Proc. VLSI Technology 2013. T190$\mathrm{T}-1912$ 